\title{
BIM 技术在给水排水工程设计中的应用研究
}

\section{Application of BIM Technology in Water Supply and Drainage Engineering Design}

\author{
王海波 \\ Haibo Wang \\ 滕州市中润供水有限公司 中国・山东 滕州 277599 \\ Tengzhou Zhongrun Water Supply Co.,Ltd., Tengzhou, Shandong, 277599, China
}

摘 要: 目前, 各种新技术不断涌现和发展。先进的建筑信息模型相关技术在建筑设计各个方面的应用越来越被建筑公司所 接受, 这可以大大改善建筑数据管理的行业问题。建筑给排水工程是 BIM 技术的一个应用领域, 论文对其应用进行了分析和 研究。

\begin{abstract}
At present, a variety of new technologies are emerging and developing. The application of advanced building information model technology in various aspects of architectural design is increasingly accepted by construction companies, which can greatly improve the industry problems of building data management. Building water supply and drainage engineering is an application field of BIM Technology. This paper analyzes and studies its application.
\end{abstract}

关键词: BIM 技术; 给水排水; 应用研究

Keywords : BIM technology; water supply and drainage; application research

DOI : 10.36012/etr.v2i8.2485

\section{1 引言}

在当今中国空前发展的今天, 人们的物质生活质量有了 飞跃, 人们对生活质量的追求也更高, 为了满足更高的要求, BIM 相关技术被应用于排水工程设计中, 确保了相关设计的 有效性和合理性。

\section{2 对 BIM 的理解}

BIM 技术是一种用于创建建筑信息模型的技术。它使用 特定建筑项目中的基本数据和信息来构建特定的建筑模型[1]。 与 $\mathrm{CAD}$ 技术相比, BIM 技术更为强大, 其固有的可视化功能 可以促进协同设计, 模拟施工过程更具有针对性, 大大提高 了工作效率, 简化了上级的相关工作。

\section{BIM 技术应用于工程设计工作}

\section{1 协同设计}

在过去的建筑设计中, 各个方面的设计师都会独立设计各自 领域的相关设计,进而掌握整体结构和设计。不同学科构成 要素的创造是独立的设计。如果不能有效地实现协同设计,
就不可能实现协同设计。另外,有必要参加第一次模拟考试。 有了扎实的专业知识,第一次模拟考试就会更顺利,工作量 也会大大提高。BIM 的应用将所有专业的设计都集中在统一 模型的构建上, 每个链接和专业设计都以统一和特定的方式 显示和反映 [1]。建立通用模型可以大大提高设计效率, 减少工 作量。BIM 在给排水设计中的应用可以很好地显示由给排水 相关数据建立的模型。在总体建筑模型中,建筑设计师可以 清楚地了解给排水设计的具体内容。有或没有专业背景的专 业设计师在调整总体方案时都可以了解相关内容。只要调整 相关的给排水参数, 整个建筑物的设计方案就可以随时调 整,这将大大促进设计的协调。

\section{2 参数化设计}

BIM 技术可以实现参数化设计。参数化设计将大大提高 模型生成和修改的速度和效率, 对开发类似设计和专用 $\mathrm{CAD}$ 软件系统具有重要意义。在以往的给排水设计中, 一些楼层 通常有类似的需要。修改后的设计需要在不同的楼层进行修 改, 增加了修改的工作量, 而且 CAD 软件的操作更为烦琐。如 
果使用 BIM, 则可以绘制建筑整体给排水的参数化设计图。

\section{3 可视化设计}

以往建筑给排水相关信息主要从设计结构相应的楼层 平面图和横断面图的不同角度进行展示。虽然这些图纸包含 所有的信息,但设计和理解这些信息是也是非常必要的。由 于其比较复杂, 需要很高的图纸审查能力, 考虑到人工三维 结构的重建不直观的, 使用 BIM 相关技术可以为更复杂的 建筑提供详细的科学图表, 可以对二维平面与三维的进行有 效结合, 具体来说, 在建筑给排水设计和图纸绘制中, 可以通 过 BIM 相关技术将平面与三维设计结合起来, 特别是对于 管道排水, 可以使其结构位置更直观, 大大提高给排水设计 的效率 ${ }^{[2]}$ 。

\section{4 管线综合设计}

如前所述, BIM 技术可以将建筑的三维空间形象化, 从 一个完整的角度展示建筑的三维空间,使设计者能够清楚地 了解建筑的空间特征和信息。对于建筑给排水, 可以合理地 进行管道敷设设计, 包括管道路径和占地图像设计, 这在 BIM 中非常重要。在测量中还可以进行三维碰撞检测, 测量 非常实用, 模型中是否存在结构冲突可以直观地显示出来, 这对于建筑给排水的相关设计尤为重要。通过对设计管线的 碰撞检测, 可以看出是否存在与其他结构的干涉和冲突。如 有干扰, 应及时调整完善方案, 进一步避免设计错误, 也可终 止设计安排留下的漏洞,给后续工程建设带来困难。

\section{4 具体问题}

在 BIM 技术的应用中, 虽然可以大大提高建筑给排水设 计的效率,但其存在的问题不容忽视。例如,管道视图的显示 范围不合理, 并且由于下水道与下水道建筑物地板有关的软 件令人困惑。它主要使用“透视深度”功能, 不仅显示地板的 管线, 还扩展相邻地板的管线, 从而导致显示地板管道的混 乱 ${ }^{[3]}$ 。针对 BIM 技术在应用中遇到的所有上述问题, 结合 BIM 技术在未来的实际应用, 本文将针对如何更好地改进 BIM 提 出一些具体的建议, 如集中精力改进与 BIM 有关的平台软件。

该平台软件的自动计算和自动绘制功能使它能够尽快 满足供水和排水的相关设计要求。该软件具有丰富的元素, 可确保其具有相对完整的信息, 开发实用功能, 并确保可以 很好地用于给排水设计中; 第三, 对于 BIM 家庭图书馆, 应 使用国家制图标准中的特定规定来补充和改进家庭图书馆,
以提高家庭图书馆的兼容性, 以便可以识别或共享不同的平 台或专业。一般来说, 在 BIM 相关技术的应用和开发中, 其 数据库集成了与工程建设相关的大量数据。这些数据支持建 筑工程的相关设计, 并且可以由各个部门共享。在未来的应 用中, 应以给排水工程建设为基础, 补充相关设计内容, 开发 数据库, 只有这样, 才能对 BIM 技术进行改进和有效补充。

其次, 它不是标准的轴测软件, 与国家/地区有关的附图 所使用的标准之间存在一定的差距, 不能直接使用。因此, 应 绘制其他给排水管道系统图纸。用 BIM 绘制给排水设计图 可以反映整体结构的相关性, 但相关性不容忽视。无法在 3D 视图中移动管道可能会阻止其他零件的显示。第三, 仍然缺 乏自动计算和辅助绘图功能, BIM 在给排水设计中会遇到一 些麻烦的环节, 如很难处理喷雾设计。如果使用其他 2D 软 件, 它将自动计算和绘制。第四, 应用程序中 BIM 系列库的 功能需要改进。结果表明, 某些家族库与不同平台的软件不 兼容, 某些设备没有相应的家族库, 或者家族库的功能不够 完善, 无法与其他软件兼容。第五, 尽管 BIM 的许多功能非 常强大且有用, 但仍需要进一步改进 BIM 的某些 2D 绘图功 能。例如, 2DCAD 工程图是工程学的工程基础, 而 BIM 相关 软件的 $2 \mathrm{D}$ 工程图格式不是 $\mathrm{CAD}$ 版本。在转换过程中, 它将 被替换或丢失, 不能直接应用于项目 ${ }^{[4]}$ 。因此, 有必要加强 $2 \mathrm{D}$ 视图格式的兼容性。

\section{5 结语}

对于建筑物来说,给排水工程的质量严重影响着人们的日 常生活, 必须注意其质量保证, 此外, 其设计的作用不可忽视, BIM 技术在其设计中的应用将有助于水供排水设计, 因此, 应 高度重视 BIM 的应用, 合理掌握和使用 BIM, 提高给排水工程 质量, 甚至提高整个项目的质量, 打造高质量的供水项目。

\section{参考文献}

[1] 裴帅.BIM 技术在建筑给水排水工程设计中的应用探讨 [J]. 城 镇建设,2020(1):164.

[2] 胡桂玲. BIM 技术在建筑给水排水工程设计中的应用探讨[J]. 砖瓦世界,2020(4): 104.

[3] 桂金, 冰琰. BI M 技术在建筑给水排水工程设计中的应用探讨 [J].商品与质量, 2020(7):90.

[4] 辛洪芹.BIM 技术在绿色建筑给排水工程中的应用研究[J].绿色 环保建材,2020,159(5):54,57. 